We collected the data by contacting the residential homes, carers, Collecting details from case notes, from the Staff nurse who made the protocol for their patients

A questionnaire based on the standards mentioned above was developed and files and prn protocols were marked against these standards.

Result. The standards from the medical file were $100 \%$ achieved. Thus indicating the importance of the psychotropic prn medication and documentation of the same.

However, the protocol that needs to be with the patient/carers had some lacuna/deficits. Overall only in $53 \%$ of the case, standards were achieved. This needs to be highlighted to the team.

The Audit gave an insight into what needs to be improved. THE FOLLOWING AREAS NEEDED IMPROVEMENT

1. There should be a prn protocol/ similar instruction to the staff about the use of prn medication(written by appropriately trained professional)

2. Prn protocol should be accessible to direct care staff

3. There should be a description of when to use the prn medication

4. There should be a description of what non-pharmacological de-escalation methods ought to be tried before using prn/ is there a detailed behaviour support plan available

5. Protocol should describe what the medication is expected to do

6. Protocol should describe the minimum time between doses if the first dose has not worked

7. Protocol should state the maximum dose in 24 hour period

8. Use of prn should be recorded

Conclusion. I hope this audit will help in improving the patient care with the right psychotropic prn medication, with correct doses and further details as mentioned in the standards of the protocol.

We also hope to ensure that in our area, prn psychotropic medication used for agitation and behavioural disturbance is used safely, appropriately and consistently by staff teams. This would be in accordance with the guidelines.

\section{Audit of the quality and content of discharge summaries from mental health inpatient units across Betsi Cadwaladr University Health Board}

Asha Dhandapani*, Sathyan Soundararajan, Laura Williams, Ediriverere Endurance Aghahowa and John Clifford

BCUHB

${ }^{\star}$ Corresponding author.

doi: 10.1192/bjo.2021.241

Aims. Our aim was to carry out an audit of summaries sent from inpatient psychiatric units across North Wales (namely Heddfan in Wrexham, Ablett in Rhyl, and Hergest in Bangor), against recommendations from 'Standards for Inpatient Mental Health Services' (RCPsych 2014) and PRSB Mental Health Discharge guidelines (2018). Method. Ablett summaries are typed onto and electronically sent through the Welsh Clinical Portal (WCP) directly to the GP. Hergest and Heddfan both have their own templates which are then sent to the GP and filed in the case notes. Data were collected from both sources. The first audit cycle used 25 discharges selected at random from the male and female open wards in each site ( $\mathrm{n}=75$ summaries). Data were collected over 3 months time using the audit proforma.

Result. All mandatory headings are automatically inputted into the WCP summary used in Ablett therefore documentation was 100\% for information such as patient name, DOB, and GP Details.
Documentation of allergies was poor across 3 sites, particularly in Hergest, in which there was no mention of allergy status in $96 \%$ of summaries. Only $13 \%$ of Ablett summaries and $0 \%$ of Hergest summaries reach the GP on the day of discharge, however, $100 \%$ of summaries from Heddfan do, possibly due to their method of 'discharge notification'. The date and location of discharge were documented in $84 \%$ of Heddfan summaries, $100 \%$ of Hergest summaries, and $100 \%$ of Ablett summaries. This implies that this heading is already incorporated into the templates for the 2 sites which scored $100 \%$. In the Ablett, medication was documented in $88 \%$, but we found that in $49 \%$ of discharge summaries, the medication was the only field filled in! In these cases, the GP may not even know why the patient had been admitted. This is clearly unacceptable. Risk history is poorly documented across the sites, with $0 \%$ in Hergest and Heddfan, and $12 \%$ in Ablett. $0 \%$ of summaries across the Health Board mentioned crisis contacts. $0 \%$ of summaries in Heddfan and Ablett contained details of the patient's care coordinator.

Conclusion. Our audit has identified a lack of psychiatry-relevant headings in the discharge summaries, particularly for those working in Ablett.

\section{Psychotropic prescribing practices in adults with} intellectual disability and autism spectrum disorder in Richmond Neurodevelpmental Services

Anushka Dissanayake ${ }^{1 \star}$, Nicholas Davey ${ }^{2}$ and Rupal Patel ${ }^{1}$

${ }^{1}$ Your Healthcare (Neurodevelopmental Services) and ${ }^{2}$ South West London St Georges Mental Health Trust

${ }^{\star}$ Corresponding author.

doi: 10.1192/bjo.2021.242

Aims. Our aim was to evaluate psychotropic prescribing practices in adults with intellectual disability (ID) and autism spectrum disorder (ASD) across the Richmond Neurodevelopmental Service (NDS).

Stopping over-medication of people with a learning disability, autism or both with psychotropics (STOMP) aims to reduce the potential harm of inappropriate use of psychotropic medications. We aimed to evaluate our prescribing practices in keeping with STOMP and the NICE guidelines.

Method. We collected information from our clinical records on patients that met the inclusion criteria $(\geq 18$ years + diagnosis of ID and autism) from October-November 2019. We gathered the following: age, sex, severity of ID, psychiatric diagnoses, psychotropic medication, presence of challenging behaviours, involvement of positive behaviour support (PBS) and documentation of a PBS plan. Result. 32 patients met our criteria (3:1 Male-Female ratio with an age range of 20-74 (Median 33 years old)). All 32 patients showed evidence of challenging behaviours. In the cohort, mild ID represented $18.8 \%(n=6)$, moderate ID $40.6 \%(n=13)$ and severe ID $40.6 \%(\mathrm{n}=13)$.

17 patients (53\%) had a PBS plan in place. For those without a PBS plan $(47 \%, n=15)$, a referral to behavioural analysis had been considered/requested in $67 \%(n=10)$.

31 patients were on psychotropic medication and $84 \%(n=26)$ had an indication documented in the notes although every patient had had a medication review in the last 6 months. $67.7 \%(\mathrm{n}=21)$ of the prescriptions were for challenging behaviours.

The average number of medications prescribed was 2 (median 2, mean 2.41) but this was reduced to 1 (median 1 , mean 1.76) when additional psychiatric diagnoses and epilepsy were excluded.

Conclusion. Prescriptions are regularly reviewed in keeping with STOMP guidance but there is more scope for utilising behaviour analysis input as well as the need to improve documentation of the rationale for psychotropic medications. 